\title{
Instrumentos de avaliação da qualidade de vida do idoso com Alzheimer: revisão integrativa da literatura
}

Alex Bacadini França ${ }^{1}$, Gabriella Santos Lima ${ }^{2}$, Sueli Marques ${ }^{3}$, Luciana Kusumota ${ }^{4}$

\footnotetext{
${ }^{1}$ Bacharel em Comunicação Social Publicidade e Propaganda, Mestre em Psicologia, Discente do Programa de PósGraduação em Psicologia, nível Doutorado, da Universidade Federal de São Carlos. São Carlos, SP, Brasil. E-mail: alex.tonante@gmail.com.

${ }^{2}$ Enfermeira. Discente do Programa de PósGraduação em Enfermagem Fundamental, nível Mestrado, da Escola de Enfermagem de Ribeirão Preto da Universidade de São Paulo. Ribeirão Preto, SP, Brasil. E- mail: gabriellasantos_3@hotmail.com.

${ }^{3}$ Enfermeira, Doutora em Enfermagem Fundamental. Professora Doutora da Escola de Enfermagem de Ribeirão Preto da Universidade de São Paulo. Ribeirão Preto, SP, Brasil. E-mail: smarques@eerp.usp.br.

${ }^{4}$ Enfermeira, Doutora em Enfermagem. Professora Doutora da Escola de Enfermagem de Ribeirão Preto da Universidade de São Paulo. Ribeirão Preto, SP, Brasil. E-mail: kusumota@eerp.usp.br.
}

Recebido: 28/10/2014.

Aceito: 03/03/2016.

Publicado: 30/06/2016.

Como citar esse artigo:

França AB, Lima GS, Marques S, Kusumota L. Instrumentos de avaliação da qualidade de vida do idoso com Alzheimer: revisão integrativa da literatura. Rev. Eletr. Enf. [Internet]. 2016 [acesso em:

____18:e1170. Disponível em: http://dx.doi.org/10.5216/ree.v18.32579.

\section{RESUMO}

O uso de instrumentos para avaliar a qualidade de vida de idosos com Alzheimer é de grande utilidade para a implementação da atenção adequada a essa população. O objetivo do estudo é buscar na literatura as evidências de validade dos instrumentos que avaliam a qualidade de vida dos idosos com Alzheimer respondido pelo próprio paciente. Trata-se de uma revisão integrativa da literatura. A busca nas bases de dados foi realizada na Pubmed, Lilacs e Cinahl. Foram selecionados 10 artigos para análise integral. Esta revisão permitiu que fosse identificado um instrumento principal de avaliação (QoL-DA), validado às diferentes populações, além da proposta de dois novos instrumentos (DQI e BASQID). A análise das propriedades de validade e consistência interna (confiabilidade) dos instrumentos foram consideradas satisfatórias. Permitiu também o fortalecimento da noção apontada pelos autores acerca da dependência entre as dimensões da qualidade de vida dos idosos e a doença de Alzheimer. Descritores: Idoso; Qualidade de Vida; Estudos de Validação; Demência; Doença de Alzheimer.

\section{INTRODUÇÃO}

A abordagem do envelhecimento populacional é recorrente nos estudos nacionais e internacionais. No ano de 2050 estima-se que existam aproximadamente dois bilhões de pessoas com 60 anos ou mais no mundo, retratando o impacto nas questões políticas, socioculturais, econômicas e demográficas que envolvem o aumento da expectativa de vida e a queda das taxas de natalidade ${ }^{(1)}$. Além do uso do termo "Expectativa de Vida" encontramos atualmente o uso frequente do termo "Qualidade de Vida" cujo conceito 
é muito antigo e ao longo da história foram criados diversos significados na busca da melhor definição.

A OMS (Organização Mundial da Saúde) considera qualidade de vida como "a percepção do indivíduo acerca de sua posição na vida, no contexto cultural e sistema de valores do local onde vive e em relação aos seus objetivos, expectativas, padrões e preocupações" ${ }^{\prime 2)}$. A multidimensionalidade do conceito qualidade de vida é relacionada com critérios biológicos, sociais e psicológicos que são encontrados no ciclo da família, nas relações sociais, ambientais, estéticas, resultando em uma junção de fatores que a sociedade considera seu padrão de conforto e bem-estar ${ }^{(3)}$.

Paradoxalmente vivemos em uma era na qual a expectativa de vida tem se elevado de maneira muito rápida, e com a mesma velocidade surgem as doenças crônicas não transmissíveis, tendo como público alvo a população contemporânea.

Encontrada com frequência em idosos, a Doença de Alzheimer (DA) leva a uma condição neurodegenerativa caracterizada por deterioração de memória e de outras funções cognitivas, comprometimento progressivo das atividades de vida diária e uma multiplicidade de alterações comportamentais e psicológicas que mais comprometem a qualidade de vida na velhice ${ }^{(4)}$. A DA é a principal causa de demência e a causa líder de incapacitação entre as pessoas com mais de 60 anos, gerando altos custos diretos e indiretos. A prevalência de demência no Brasil foi estimada em 390.000 casos em 2000, sendo cerca de $50 \%$ destes por $\mathrm{DA}^{(5)}$.

Dessa forma, a elaboração de instrumentos técnicos que permitam a avaliação de características da população deve atender a certas condições e requerem procedimentos padronizados. Entretanto, também é necessário que sejam evidenciadas e analisadas suas propriedades psicométricas. Visto que um instrumento que não evidencia tais propriedades é impedido de ser reconhecido cientificamente ${ }^{(6)}$.

Como propriedades psicométricas, destacam-se a precisão (fidedignidade) e a validade. A precisão pode ser entendida como a consistência ou estabilidade, definida como a extensão em que as medidas são replicáveis, seja por um mesmo examinador ou por diferentes examinadores, fazendo a mesma medida de um construto. Para isso, usualmente, utilizam-se dois meios: o coeficiente de correlação produto-momento de Pearson e o coeficiente alpha de Cronbach, em que se calcula a correlação de cada item com todos os demais, indicando uma correlação média entre as variáveis ${ }^{(7)}$.

Merecem destaque as evidências referentes à validade que pode ser classificada em quatro amplas categorias: a) evidências de validade baseadas na análise do conteúdo ou domínio; b) evidências de validade baseadas nas relações com variáveis externas; c) evidências baseadas na estrutura interna; d) evidências baseadas no processo de resposta. Diante disso, de acordo com uma boa escala é aquela que permite uma fácil aplicação, pontuação e interpretação, além de tomar o menor tempo possível, tanto do examinador quanto do examinado ${ }^{(8)}$. Ela também deve ser relevante e apropriada para a amostra de interesse e essencialmente, deve ter indicativos de precisão e de validade coerentes com os objetivos que se pretende alcançar $^{(9)}$.

Nesse contexto e a fim de nortear futuras avaliações, o presente estudo teve como objetivo buscar na 
literatura as evidências de validade dos instrumentos elaborados para avaliar a qualidade de vida dos idosos com Alzheimer.

\section{MÉTODO}

Para o desenvolvimento desse estudo optou-se pela revisão integrativa da literatura. Esse método permite sintetizar de forma sistematizada o conhecimento sobre um determinado assunto, possibilitando a implementação dos resultados significativos dos estudos na prática assistencial. Atualmente há necessidade de garantir uma prática assistencial baseada em evidências científicas e a revisão integrativa tem sido assinalada como uma ferramenta essencial dentro dessa área ${ }^{(10)}$.

A Prática Baseada na Evidência (PBE) trata-se da busca de evidências mais fidedignas e atualizadas sobre um problema que pode abranger, por exemplo, a assistência em saúde, contexto clínico e habilidade profissional que contribuíram para o aperfeiçoamento da prática clínica e da tomada de decisão ${ }^{(11)}$.

Assim, a metodologia da revisão integrativa da literatura é uma abordagem que permite a inclusão de metodologias diversificadas tendo um papel maior na prática baseada em evidências, auxiliando o pesquisador sumarizar literatura teórica e empírica sobre um tema específico ${ }^{(12)}$.

No presente estudo, optou-se por adotar para elaboração da questão norteadora, a estratégia PICO, que trata de acrônimo para Paciente, Intervenção, Comparação e Outcomes ou desfecho ${ }^{(13)}$. Os termos utilizados nesta pesquisa foram: "P" Idosos com Alzheimer; "I" Validação e Precisão; "C" os autores não têm objetivo de comparar intervenções; "O" Instrumentos de Qualidade de Vida. Assim foi definida a questão norteadora: "Quais as evidências de validade e precisão apresentadas pelos instrumentos que avaliam a qualidade de vida do idoso com Alzheimer?".

O estudo foi desenvolvido na Escola de Enfermagem de Ribeirão Preto (EERP-SP), no primeiro bimestre de 2016. As bases de dados utilizadas foram National Library of Medicine National Institutes of Health (PUBMED), Cumulative Index to Nursing \& Allied Health Literature (CINAHL) e Literatura Latino-americana e do Caribe em Ciências da Saúde (LILACS). Os descritores controlados foram extraídos do Medical Subject Headings (MeSH) e Descritores em Saúde (DeCS), sendo incluídos também os descritores não controlados no intuito de ampliar os achados na literatura. O operador booleano "AND" foi combinado com os descritores resultando nas seguintes equações de busca: 1) Pubmed - (((("Aged"[Mesh]) AND "Validation Studies" [Publication Type]) AND "Quality of Life"[Mesh]) AND dementia) AND "Alzheimer Disease"[Mesh]; ((((aged) AND validation studies) AND quality of life) AND dementia) AND alzheimer's disease; 2) CINAHL - aged AND validation studies AND quality of life AND dementia AND alzheimer's disease; 3)Lilacs - ("Idoso") AND "Qualidade de vida" [Palavras] and ("Estudos de validacao") AND "Demencia" [Palavras] and "Doenca de alzheimer" [Palavras].

Foi definido o intervalo de janeiro de 2006 a fevereiro de 2016 (últimos 10 anos) visto que as propriedades psicométricas de um teste devem ser revisadas periodicamente, no prazo máximo de 10 anos entre um estudo e outro ${ }^{(14)}$. Consideramos para análise dos artigos somente os que estavam no idioma 
português e inglês, disponíveis na íntegra e no modo on-line.

Os artigos foram avaliados por meio de leitura, na íntegra, dos textos, que deveriam responder aos seguintes critérios de inclusão: estudos publicados nos últimos 10 anos, redigidos no idioma português e inglês com as temáticas relacionadas à validade e precisão apresentadas pelos instrumentos que avaliam a qualidade de vida do idoso com Alzheimer, respondido pelos próprios idosos.

A análise foi conduzida por meio de um quadro sinóptico elaborado pelos próprios autores que contemplou as seguintes categorias: título do artigo, periódico onde foi publicado, ano de publicação, nome do primeiro autor, filiação do primeiro autor, objetivo do estudo, amostra de participantes, país de origem da amostra, grau de demência dos participantes, apresentação das evidências de validade e precisão dos instrumentos e a base de dados em que estava inserido. Na sequência, foram agrupados em duas categorias: novos instrumentos e adaptação cultural.

Por sua vez, a apresentação dos resultados e discussão dos dados obtidos foi feita de forma descritiva, almejando que o leitor capture a profundidade e a amplitude do tema, e suscitar um novo entendimento do fenômeno de preocupação(15).

Por meio das estratégias de busca e critérios de elegibilidade, foram localizados 30 artigos na busca às bases de dados. Após a leitura dos títulos, resumos e íntegra foram incluídos para responder a questão de pesquisa 10 artigos, sendo três da CINAHL, seis da PUBMED e um da LILACS.

Na Figura 1, é apresentado o processo de seleção dos estudos, conforme recomendação PRISMA statement (Preferred Reporting Items for Systematic Reviews and Meta-Analysis) ${ }^{(16)}$. 


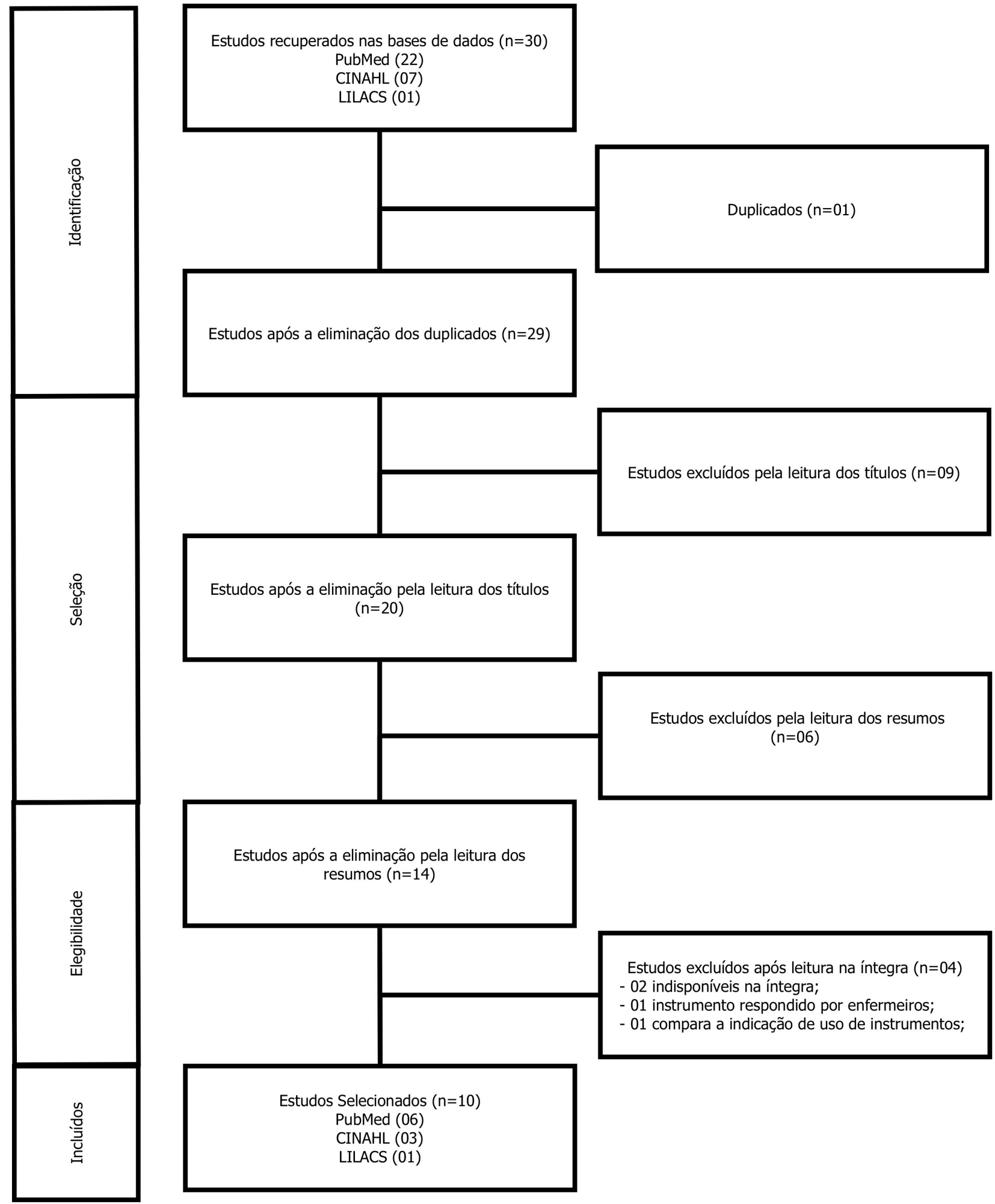

Figura 1: Processo de seleção dos estudos (Fluxograma PRISMA statement). Ribeirão Preto-SP,2016.

\section{RESULTADOS}

A síntese dos resultados obtidos é apresentada no Quadro 1, baseando-se em um instrumento validado contendo as seguintes características dos artigos: número da referência, país de origem, ano de publicação, bases de dados, instrumento utilizado para aferir demência, grau da demência, objetivos e características do estudo (validade/precisão) ${ }^{(17)}$. 
Pode-se constatar que, em sua maioria, as publicações encontradas se originaram em países europeus

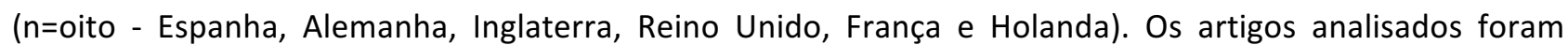
publicados entre os anos de 2006 a 2012. Dentre as categorias estabelecidas neste estudo, todos os artigos mencionavam a validade e precisão de instrumentos que avaliam a qualidade de vida do idoso com Alzheimer. Destaca-se ainda que a maioria dos artigos era no idioma inglês ( $n=n o v e)$. Durante o processo de seleção dos estudos foram excluídos 20 artigos, que não responderam à pergunta norteadora e/ou não atenderam aos critérios de inclusão.

Para realização da síntese, os estudos selecionados foram classificados em duas categorias, de acordo com o tipo de desenvolvimento proposto: 1) Novos instrumentos e 2) Adaptação à população específica. 
Quadro 1: Distribuição dos artigos incluídos na revisão integrativa. Ribeirão Preto, SP, Brasil, 2016.

\begin{tabular}{|c|c|c|c|c|c|}
\hline $\begin{array}{c}\text { Autores/Periódico/Ano } \\
\text { publicação/ País }\end{array}$ & $\begin{array}{l}\text { Base de } \\
\text { Dados }\end{array}$ & Instrumento (s) Utilizado (s) & $\begin{array}{c}\text { Grau de } \\
\text { Demência }\end{array}$ & Objetivo (s) & *Resultados de Validade e Precisão \\
\hline $\begin{array}{l}\text { A1. Schölzel-Dorenbos, Arons, } \\
\text { Wammes, Rikkert, Krabbe/ } \\
\text { Health and Quality of Life } \\
\text { Outcomes /2012 /Holanda }\end{array}$ & Pubmed & DSM-IV-TR; Clinical Dementia Rating & $\begin{array}{l}\text { Muito Leve a } \\
\text { Moderada }\end{array}$ & $\begin{array}{l}\text { Desenvolver e validar o } \\
\text { protótipo de uma medida } \\
\text { específica de índice de } \\
\text { demência DQI }\end{array}$ & $\begin{array}{l}\text { Validade de conteúdo: juízes avaliadores; } \\
\text { Validade concorrente feita por correlação } \\
\text { entre os domínios da DQI e EQ-5D+C: } \\
\text { estatisticamente significantes para pacientes e } \\
\text { cuidadores: memoria, cognição, orientação, } \\
\text { independência, auto-cuidado, atividades } \\
\text { diárias, humor, depressão/ansiedade }\end{array}$ \\
\hline $\begin{array}{l}\text { A2. Trigg, Skevington, Jones/ The } \\
\text { Gerontological Society of } \\
\text { America/2007 / Inglaterra }\end{array}$ & Pubmed & MEEM; GDS-15 & $\begin{array}{l}\text { Leve a } \\
\text { Moderada }\end{array}$ & $\begin{array}{l}\text { Desenvolver uma medida de } \\
\text { avaliação de qualidade de } \\
\text { vida subjetiva de pessoas com } \\
\text { ligeira a moderada demência }\end{array}$ & $\begin{array}{l}\text { Validade de conteúdo: entrevistas } \\
\text { qualitativas; BASQID: } \alpha=0.89 \text {; validade } \\
\text { concorrente: BASQID x GDS-15: } r=0.58\end{array}$ \\
\hline $\begin{array}{l}\text { A3. Wolak, Novella, Drame, } \\
\text { Guillemin, Di Pollina, Ankri, et al/ } \\
\text { Aging\& Mental Health/2009/ } \\
\text { França }\end{array}$ & Pubmed & DSM IV; MEEM; Duke Profile & $\begin{array}{l}\text { Leve a } \\
\text { moderada }\end{array}$ & $\begin{array}{l}\text { Avaliar as propriedades } \\
\text { psicométricas e adaptação } \\
\text { transcultural (QoL-AD) para } \\
\text { Francês }\end{array}$ & $\begin{array}{c}\text { Consistência interna: sub escala paciente e } \\
\text { cuidador } \alpha=0.70 \text { / Validade convergente QoL- } \\
\text { AD x Duke Profile: } 0.25<r<0.54 \text { para pacientes } \\
\text { e } 0.25<r<0.39 \text { para cuidadores }(p<0.05)\end{array}$ \\
\hline $\begin{array}{l}\text { A4. Matsui, Nakaaki, Murata, } \\
\text { Sato, Shinagawa, Tatsumi, et al/ } \\
\text { Dementia and Geriatric } \\
\text { Cognitive Disorders/ 2006/ } \\
\text { Japão }\end{array}$ & CINAHL & QOL-AD;NPI;HADL;SMQ & $\begin{array}{l}\text { Leve a } \\
\text { moderado }\end{array}$ & $\begin{array}{c}\text { Desenvolver a versão } \\
\text { japonesa da QOL-AD e } \\
\text { verificar sua confiabilidade e } \\
\text { fatores que alteram a } \\
\text { qualidade de vida dos } \\
\text { portadores de Alzheimer }\end{array}$ & $\begin{array}{l}\text { Consistência interna: sub escala paciente } \\
\alpha=0.85 \text { e sub escala cuidador: } \alpha=0.82 \\
\text { Nalidade concorrente a partir dos escores } \\
\text { pacientes e cuidadores: } r=0.60, p<0.01\end{array}$ \\
\hline $\begin{array}{l}\text { A5. Gómez-Gallego, Gómez- } \\
\text { Amorb, Gómez-Garcíac/ } \\
\text { Neurología/2011/ Espanha }\end{array}$ & Pubmed & MEEM; HUI-3 & $\begin{array}{l}\text { Leve a } \\
\text { moderado }\end{array}$ & $\begin{array}{l}\text { Determinar as propriedades } \\
\text { psicométricas da versão } \\
\text { espanhola da escala QoL-AD } \\
\text { em pacientes com Alzheimer, } \\
\text { Cuidadores e Profissionais. }\end{array}$ & $\begin{array}{l}\text { Consistência interna: sub escala paciente } \\
\alpha=0.85 \text {, sub escala cuidador } \alpha=0.84 \text {, sub escala } \\
\text { profissional } \alpha=0.9 \text {. Validade concorrente } \\
\text { (escore total): QoL-Ad x HUI-3: }-0.47\end{array}$ \\
\hline $\begin{array}{l}\text { A6. Novelli, Nitrinia, } \\
\text { Caramellia/Aging \& Mental } \\
\text { Health/2010/ Brasil }\end{array}$ & CINAHL & $\begin{array}{l}\text { QV-AD/PQOL/CPQOL/WHOQOL } \\
\text { breve/CQOL }\end{array}$ & $\begin{array}{l}\text { leve ou } \\
\text { moderada }\end{array}$ & $\begin{array}{l}\text { Avaliar a confiabilidade e } \\
\text { validade da construção da } \\
\text { escala (QoL-AD) versão } \\
\text { brasileira }\end{array}$ & $\begin{array}{l}\text { Validade de construto: QoL-AD x WHOQOL- } \\
\text { breve } r>0.70\end{array}$ \\
\hline
\end{tabular}




\begin{tabular}{|c|c|c|c|c|c|}
\hline $\begin{array}{l}\text { Autores/Periódico/Ano } \\
\text { publicação/ País }\end{array}$ & $\begin{array}{l}\text { Base de } \\
\text { Dados }\end{array}$ & Instrumento (s) Utilizado (s) & $\begin{array}{c}\text { Grau de } \\
\text { Demência }\end{array}$ & Objetivo (s) & *Resultados de Validade e Precisão \\
\hline $\begin{array}{l}\text { A7. Salas, Logsdon, Olazara'n, } \\
\text { Martınez-Martın, Msu-Adru/ } \\
\text { Aging \& Mental Health/2011/ } \\
\text { Espanha }\end{array}$ & Pubmed & $\begin{array}{c}\text { QOL-Adp/QOL- } \\
\text { Adc/MMSE/GDS/CSDD/QUALID/EQ- } \\
\text { VAS/LI/BI/AI/NPI }\end{array}$ & & $\begin{array}{c}\text { Avaliar os atributos } \\
\text { psicométricos da versão em } \\
\text { espanhol da (QoL-AD) em } \\
\text { pacientes institucionalizados } \\
\text { e cuidadores familiares na } \\
\text { Espanha }\end{array}$ & $\begin{array}{c}\text { Consistência interna: sub escala paciente } \\
\alpha=0.90 \text {, sub escala cuidador } \alpha=0.86 \text {; Validade } \\
\text { convergente: Qol-AD x QUALID:0.43 e Qol-AD } \\
\text { x EQD-5: } 0.65\end{array}$ \\
\hline $\begin{array}{c}\text { A8. Hoe, Katona, Roch, } \\
\text { Livingston/ Age and Ageing/ } \\
\text { 2005/ Inglaterra }\end{array}$ & CINAHL & MMEE & $\begin{array}{l}\text { Dêmencia } \\
\text { Severa } \\
(\text { MEEM<12) }\end{array}$ & $\begin{array}{c}\text { Examinar a utilidade da escala } \\
\text { (QoL-AD) em pessoas com } \\
\text { demência grave } \\
\text { e seus cuidadores, } \\
\text { verificando a validade de } \\
\text { construto e consistência } \\
\text { interna. }\end{array}$ & $\begin{array}{l}\text { Consistência interna } \alpha=0.78 \text { quando } \\
\text { considerado instrumento todo }\end{array}$ \\
\hline $\begin{array}{l}\text { A9. Voigt-Radloff, Leonhart, } \\
\text { Schutzwohl, Jurjanz, Reuster, } \\
\text { Gerner, et al/ European Journal } \\
\text { of Neurology/2012/ Alemanha }\end{array}$ & Pubmed & MEEM/ ADAS-cog/ CSDD/IDDD & $\begin{array}{l}\text { Leve a } \\
\text { Moderada }\end{array}$ & $\begin{array}{c}\text { Traduzir o instrumento } \\
\text { (DQOL) para o alemão e } \\
\text { avaliar a sua construção e } \\
\text { validade concorrente em } \\
\text { comunidade de pessoas com } \\
\text { demência }\end{array}$ & $\begin{array}{l}\text { Consistência interna } \alpha=0.61 \text { a } 0.84 \text { por fator. } \\
\text { Validade concorrente DQoL x SF-12: } \\
\qquad r=>0.43>r-0.63\end{array}$ \\
\hline $\begin{array}{l}\text { A10. Novelli, Rovere, Nitrini, } \\
\text { Caramelli/Arquivo } \\
\text { Neuropsiquiatria. 2005/Brasil }\end{array}$ & Lilacs & MEEM/DSM III-R-14 & $\begin{array}{l}\text { Leve a } \\
\text { Moderada }\end{array}$ & $\begin{array}{c}\text { Apresentar a validação } \\
\text { interna da escala (QV-AD) } \\
\text { para pacientes com doença } \\
\text { de Alzheimer ( } A D \text { ) e os seus } \\
\text { membros cuidadores/ família }\end{array}$ & $\begin{array}{l}\text { Consistência interna: sub escala paciente } \\
\alpha=0.81 \text { e sub escala cuidador } \alpha=0.85 \text {. }\end{array}$ \\
\hline
\end{tabular}

de critério e validade de construto. Estes itens estão detalhados ao longo do texto. 
Novos instrumentos

A1 - Estudo de validação do protótipo de uma medida de índice de doenças específicas para qualidade de vida relacionada à saúde em demência (Validation study of the prototype of a disease-specific index measure for health-related quality of life in dementia)

Neste estudo realizado na Holanda ${ }^{(18)}$, desenvolveram uma estrutura, para elaboração do instrumento denominado Dementia Quality of Life Instrument (DQI), baseada em: revisão da literatura, entrevistas qualitativas com pessoas com demência e seus cuidadores, a opinião de especialistas e discussão da equipe. A validação semântica do instrumento foi feita por meio da avaliação de 241 profissionais da saúde, que julgaram e classificaram a relevância dos itens e dos domínios elaborados. Após esta etapa, foi realizado um estudo transversal com indivíduos com demência de muito leve a moderada e seus cuidadores $(N=145)$ para avaliar a viabilidade e a validade concorrente com outros instrumentos de saúde genéricos. Em relação à análise semântica, os profissionais julgaram todos os domínios e itens relevantes. As diferenças nas correlações simultânea com os instrumentos do estado de saúde genéricos implica que os domínios específicos de demência do DQI realmente forneceram informações diferentes, o que era esperado.

A2 - Como podemos melhorar a avaliação da qualidade de vida das pessoas com demência? $\mathrm{O}$ instrumento de avaliação de qualidade de vida subjetiva nas pessoas com demência da Universidade de Bath (How Can We Best Assess the Quality of Life of People With Dementia? The Bath Assessment of Subjective Quality of Life in Dementia (BASQID)) $)^{(19)}$

Este artigo traz evidências sobre as propriedades psicométricas do instrumento de autorrelato BASQID, incluindo a relação com outros indicadores clínicos, tais como a depressão e a cognição. O BASQID contém 14 itens que avaliam uma série de questões de qualidade de vida a partir da perspectiva da pessoa com demência. Incluiu o desenvolvimento qualitativo de um quadro conceitual, gerado a partir de entrevistas em profundidade com 30 pessoas com demência em estado leve a moderado. Os autores exploraram a própria concepção dessas pessoas sobre qualidade de vida e a forma em que a demência afeta essa qualidade de vida. Os resultados mostraram que a BASQID pode ser divida em dois componentes: componente 1 representou uma subescala de Satisfação de vida e o componente 2 representou uma subescala de Sentimentos positivos de qualidade de vida. A pontuação no BASQID mostrou moderada correlação com a GDS-15 ( $r=0.58$ ) e baixa a moderada associação com o WHOQOL-BREF ( $r=0.32 ; \alpha=0.59)$. A consistência interna do BASQID foi calculada por meio de alfa de Cronbach $(\alpha=0,89 ; n=143)$, indicando boa consistência interna total. Pode-se dizer que o instrumento traz bons índices de precisão e de validade. 
A3 - Adaptação transcultural e validação psicométrica da versão francesa da QoL- AD (Transcultural adaptation and psychometric validation of a French-language version of the QoL-AD) ${ }^{(20)}$

O objetivo geral do estudo foi conduzir uma adaptação cultural e a validação psicométrica do instrumento Quality of Life in Alzheimer (Qol - AD). O QoL-AD inclui a avaliação do cuidador em relação ao paciente e a avaliação do próprio paciente de si. O procedimento utilizado foi conduzido com o consentimento e aprovação da autora da versão original em inglês do instrumento. Participaram do estudo 102 pacientes e seus cuidadores e mais 25 profissionais de saúde. A versão francesa da QoL-AD apresentou boa consistência interna $(\alpha=0.70)$ e boa confiabilidade $(r=0.80)$ em um intervalo de duas semanas. Validade convergente entre a QoL-AD e as dimensões do Perfil de Saúde de Duke também foi considerada boa.

A4 - Determinantes da Qualidade de Vida em pacientes da doença de Alzheimer, avaliada pela versão japonesa da Qualidade de Vida - Escala da doença de Alzheimer (Determinants of the Quality of Life in Alzheimer's Disease Patients as Assessed by the Japanese Version of the Quality of Life-Alzheimer's Disease Scale) $)^{(21)}$

Foi realizada a validação cultural e analisada as propriedades psicométricas da versão japonesa do Quality of Life in Alzheimer (QoL-AD). A tradução do instrumento para a língua japonesa foi realizada seguindo padrões de retro-tradução, na qual a versão original em inglês foi traduzida para japonês e depois retraduzida para o inglês por pessoas independentes da primeira versão, sendo que o autor original participou da avaliação semântica a fim de apontar alguma discrepância na retro-tradução. Após a validação semântica, o instrumento foi aplicado na amostra de pacientes japoneses idosos e aos cuidadores. A amostra final do estudo ficou composta por 140 idosos japoneses com Alzheimer leve a moderado e seus respectivos cuidadores. $O$ índice de confiabilidade para os pacientes foi $\alpha=0.84$ e os cuidadores obtiveram $\alpha=0,82$. $O$ coeficiente de correlação intra-classe foi de 0.84 para pacientes e 0.91 para os cuidadores. A confiabilidade teste-reteste $(n=83)$ da pontuação total após um mês foi excelente (pacientes: $\alpha=0.84$ e cuidadores: $\alpha=0.91$ ). A validade concorrente obteve índice de concordância significativo na pontuação total determinada a partir das respostas dos pacientes e dos cuidadores respostas $(r=0.60, p<0,01)$.

A5 - Validação da versão espanhola da escala de QoL em pacientes com Alzheimer seus cuidadores e Profissionais da Saúde (Validation of the Spanish version of the QoL-AD scale in Alzheimer disease patients, their carers, and health professionals) ${ }^{(22)}$

Este estudo teve como objetivo aferir a validade psicométrica da versão em espanhol do instrumento Quality of Life in Alzheimer (QoL-AD) em pacientes, cuidadores e profissionais de saúde. A amostra foi composta por 102 pacientes e seus cuidadores além de 25 profissionais de saúde. Os índices de confiabilidade obtidos foram: Pacientes $\alpha=0.85$, Cuidadores $\alpha=0.84$, Profissionais $\alpha=0.91$. A validade de 
construto foi realizada por meio de análise de componentes principais e obtido três fatores com valores próprios superiores a um (1), que explicaram $61,30 \%$ da variância total. Os fatores obtidos apresentaram boa consistência interna $(\alpha \geq 0.70)$ e foram interpretados como segue: fator de saúde $\left(r^{2}=29,57 \%\right)$, relações sociais e fator de ambiente $\left(r^{2}=17,27 \%\right)$ e fator de capacidade funcional $\left(r^{2}=14,46 \%\right)$.

A6 - Validação da versão brasileira da escala de qualidade de vida para pacientes com Doença de Alzheimer e seus cuidadores QoL-AD (Validation of the Brazilian version of the quality of life scale for patients with Alzheimer's disease and their caregivers - QoL-AD) $)^{(23)}$

Este artigo traz como objetivo avaliar a confiabilidade e a validade da versão brasileira da escala de qualidade de vida na doença de Alzheimer. O estudo foi conduzido no Hospital das Clínicas de São Paulo com autorização prévia da autora da versão original do instrumento. Participaram 60 idosos com Alzheimer leve a moderado e seus cuidadores $(\mathrm{N}=120)$. A validade de conteúdo foi avaliado por especialistas e pesquisadores durante a fase de concepção. A confiabilidade para a versão dos pacientes foi de 0.80 e para os cuidadores foi de 0.83 . A pontuação total da escala de QoL-AD brasileira mostrou correlações significativas com a pontuação total do WHOQOL-breve, um instrumento genérico de avaliação de qualidade de vida. Os coeficientes de correlação foram superiores a 0.70 em todas as três versões da escala.

A7 - Propriedades psicométricas do QoL-AD Espanhol em pacientes institucionalizados com demência e seus cuidadores familiares na Espanha (Psychometric properties of the Spanish QoL-AD with institutionalized dementia patients and their Family caregivers in Spain) ${ }^{(24))}$

Este estudo teve como objetivo a avaliação dos atributos psicométricos da versão em espanhol da Escala de Qualidade de Vida - DA em pacientes institucionalizados e seus cuidadores familiares na Espanha. A amostra total ficou composta por 101 pacientes institucionalizados com diagnóstico de doença de Alzheimer (DA) e seus cuidadores $(N=202)$. Sendo que $81,2 \%$ dos pacientes apresentavam demência tipo Alzheimer e 18,8\% tinham demência mista (DA juntamente com doenças cerebrovasculares). A deterioração cognitiva constatada foi desde a deterioração cognitiva moderada até a deterioração cognitiva grave. Dos 101 pacientes iniciais, 71 pacientes não entenderam ou não quiseram colaborar para completar a QoL-AD versão paciente. A consistência interna determinada por meio do índice de alpha de Cronbach foi de 0.86 para a QoL-AD versão cuidadores e 0.90 para a QoL-AD versão pacientes. A correlação item-total corrigido variou entre 0.11 (dinheiro) para 0.67 (vida em geral) na QoL-AD versão cuidadores e na QoL-AD versão pacientes variou entre 0.28 (memória) e 0.84 (energia).

A8 - Uso da QoL-AD para mensurar qualidade de vida nas pessoas com demência severa - O estudo LASER$A D$ (Use of the QoL-AD for measuring quality of life in people with severe dementia-the LASER-AD study) ${ }^{(25)}$

Este foi o primeiro estudo a reportar evidências de validade e precisão do QoL-AD em pessoas com escore entre 3 e 11 avaliadas por meio do Mini Exame do Estado Mental. Os autores justificam este ponto 
de corte por duas razões: este era o grupo em que não haviam medidas padronizadas validadas para QoL-AD sendo também o grupo em que o uso de inibidores de colinesterase não são recomendados. A validade de construto do instrumento foi avaliada pela correlação da escala com medidas de cognição, humor, sintomas neuropsiquiátricos, atividades da vida diária entre outros. A correlação item-total entre cada item QoL-AD e a pontuação total do QoL-AD variou entre 0.35 e 0.81 . O alpha de Cronbach para o instrumento todo foi de 0.78. Todavia, a escala é inapropriada para as pessoas com escores abaixo de três avaliados pelo MEEM.

A9 - Instrumento de Qualidade de Vida na Demência - construção e validade concorrente pacientes com demência leve a moderada (Dementia quality of life instrument - construct and concurrent validity in patients with mild to moderate dementia) ${ }^{(26)}$

O objetivo principal deste estudo foi traduzir o instrumento de qualidade de vida (Dementia quality of life instrument - DQoL ) para o alemão e avaliar a sua construção e validade concorrente em comunidade de pessoas com demência ligeira a moderada. A tradução inicial foi realizada por um psicólogo e um terapeuta ocupacional que traduziram as instruções, os itens e os formatos de respostas a partir de uma versão holandesa para o alemão, considerando também a versão em inglês. Após as traduções foram realizados testes piloto com a população-alvo. Em seguida, foram testados os modelos por meio de análise fatorial confirmatória e verificados os índices de consistência interna. Os autores encontram boa consistência interna ( $\alpha=0.6$ e 0.8 ) e correlações moderadas entre o instrumento e escalas de qualidade de vida (coeficiente de Spearman entre 0.3 e 0.6). A análise da validade concorrente revelou correlações moderadas $(r>0.43)$ com a Escala Mental SF-12 e o escore de QoL-AD. Efeito negativo foi moderadamente associado apenas com o SF12 na Escala Mental ( $r>0.63$ ). Por fim, a análise fatorial confirmatória indicou um mau ajuste do modelo na amostra utilizada ( $n=286 ; x^{2}=888.6$, g.I. $\left.=391, p<0.001\right)$.

A10 - Adaptação transcultural da escala de qualidade de vida para pacientes com Doença de Alzheimer (Cross-cutural adaptation of the quality of life assessment scale on Alzheimer disease) $^{(27)}$

Os autores brasileiros apresentam os dados de validação interna da escala de qualidade de vida para pacientes com doença de Alzheimer e seus respectivos cuidadores. A escala foi adaptada seguindo método que envolveu a tradução do instrumento original em inglês para o português, após as devidas análises, o instrumento passou por uma retrotradução (português-inglês) e avaliações de equivalência. Os índices de correlação encontrados na avaliação intra-examinador foram 0,87/0,95/0,95 $(p<0,001)$ para as versões do paciente, do familiar e do cuidador, respectivamente. Para a verificação da consistência interna, o instrumento adaptado foi administrado em 40 pacientes com doença de Alzheimer de intensidade leve a moderada. A confiabilidade para as versões do paciente e do familiar foram $\alpha=0.81$ e 0.85 , respectivamente, e com relação a $Q V$ do cuidador $\alpha=0.84$. 


\section{DISCUSSÃO}

Esta revisão permitiu identificar que os instrumentos que buscam avaliar a qualidade de vida do paciente idoso com Alzheimer incluíram aspectos físicos, emocionais, sociais, econômicos, relação com a família e com a equipe de saúde. Entende-se que, para que sejam adotadas medidas de intervenção, esses aspectos devem ser compreendidos e identificados de forma geral e assim implementar medidas para a melhora da qualidade de vida. Porém, ressalta-se que apenas dois dentre os 10 estudos analisados pretenderam desenvolver novos instrumentos de avaliação (Dementia Quality of life Instrument- DQI ${ }^{(18)}$ e o The Bath Assessment of Subjective Quality of Life in Dementia (BASQID)) ${ }^{(19)}$.

O Dementia Quality of life Instrument (DQI) ${ }^{(18)}$ representa um esforço de contribuir para a investigação da qualidade de vida junto à população idosa acometida por demência e também colaborar para o enriquecimento da área de avaliação. Vale ressaltar que o processo de elaboração do DQI foi embasado na revisão da literatura, entrevistas qualitativas com pessoas com demência e seus cuidadores, opinião de especialistas e discussão da equipe. A investigação das evidências de validade de conteúdo, realizada por especialistas, é importante para o aprimoramento da versão original de um instrumento. Isto porque os juízes realizam sugestões que facilitaram a compreensão dos itens por parte da população alvo, evitando possíveis equívocos dos participantes. Além disso, podem auxiliar na seleção dos melhores itens para compor a escala ${ }^{(28-29)}$. Os autores colocam que o protótipo DQI pode ser considerado válido e viável para pacientes e cuidadores, sendo apropriado para a demência de leve a moderada. Entretanto, reforçam que são necessárias mais pesquisas para gerar valores para cada uma das possíveis dimensões que o DQI avalia.

O instrumento de avaliação de qualidade de vida subjetiva nas pessoas com demência da Universidade de Bath traz grandes contribuições ao utilizar entrevistas em profundidade como meio de criação dos itens, obtendo bons índices de precisão e de validade ao final. Todavia, ainda requer novos estudos para confirmar a estrutura fatorial encontrada.

Em relação à categoria adaptação à população específica, oito dos 10 estudos visaram a adaptação transcultural e validação psicométrica do instrumento Quality of Life - Alzheimer Disease. Observou-se que todos os estudos encontrados foram realizados com consentimento da autora original, Rebecca Longsdon. Os estudos dentro dessa categoria ( $A 3, A 4, A 5, A 6, A 7, A 8, A 9$ e $A 10)$ também demonstraram seguir as diretrizes propostas pelo International Test Commission ${ }^{(30)}$, que visa unir diferentes práticas internacionais a fim de promover a prática adequada na adaptação de instrumentos de avaliação nos diversos contextos culturais. Além de possuir guias para facilitar a realização de estudos que visam verificar as evidências de validade e precisão de instrumentos. Neste contexto, pode-se afirmar que os artigos que visaram à adaptação transcultural e validação psicométricas apresentaram métodos de coleta e técnicas de análise de dados semelhantes entre si. A verificação da consistência interna (precisão) foi feita por meio do cálculo de alpha de cronbach. As versões dos instrumentos elaborados a partir da QoL - AD apresentaram bons índices de consistência interna, visto que apresentaram alpha de Cronbach satisfatórios nas duas versões, paciente e cuidador (França $\alpha=0,70$; Espanha $\alpha=0,84$ e 0,85 ; Japão $\alpha=0,82$ e 0,84; Brasil $\alpha=0,80$ e 0,83$)^{(18-26)}$. 
O estudo A6 (Propriedades psicométricas do QoL-AD Espanhol em pacientes institucionalizados com demência e seus cuidadores familiares na Espanha) foi inserido nesta mesma categoria por se tratar da aplicação do instrumento adaptado no estudo A4 (Validação da versão espanhola da escala de QoL em pacientes com Alzheimer, seus cuidadores e Profissionais da Saúde) em idosos institucionalizados com Alzheimer. O único que utilizou como amostra, pacientes com diagnóstico de demência mista (DA juntamente com doenças cerebrovasculares) além de deterioração cognitiva de nível moderada até grave, apresentando características diferentes daquela da população utilizada na adaptação transcultural. $\mathrm{O}$ autor relata que dos 101 pacientes iniciais, 71 pacientes não entenderam ou não quiseram colaborar para completar a QoL-AD versão paciente. Tal fato pode ter ocorrido devido à gravidade da demência, demonstrando que os instrumentos que visam avaliar a qualidade de vida em idosos com Alzheimer devem levar em conta o grau/estágio da patologia ${ }^{(31-32)}$. Neste contexto, com a impossibilidade de o paciente responder o instrumento em decorrência do grau em que se encontra a demência, a utilização de um instrumento com o intuito de mensurar a qualidade de vida em idosos com Alzheimer deve ser feita com cuidado. Por outro lado, é importante e necessário investigar idosos sem comprometimento cognitivo avançado, atentando para as características da subjetividade no constructo da QV, as quais são multifatoriais e complexas, relacionadas aos aspectos econômicos, socioculturais, à experiência pessoal e estilos de vida. A exploração inicial da qualidade de vida de idosos com Alzheimer por meio de instrumentos padronizados evidencia indicativos aos profissionais, justificada pela possibilidade do paciente relatar, sob sua perspectiva, o que, de fato, lhe acontece e o que sente.

Vale ressaltar que outro autor abordou a comparação de dois questionários, trouxe contribuições importantes em relação a dados psicométricos dos dois principais questionários que se propõem a mensurar a qualidade de vida em idosos com Alzheimer, o DQoL e o QoL-AD ${ }^{(33)}$. Os autores compararam as propriedades psicométricas em 123 pacientes com idades a partir de 65 anos que sofrem da doença de Alzheimer. Os dois apresentaram boa reprodutividade baseada em índices de correlação interclasses com intervalo de duas semanas entre as aplicações $(r=0.80)$ e boa consistência interna avaliada por meio de alfa de Cronbach $(\alpha=0.70)$. Além disso, os autores concluíram que para avaliações mais rápidas, como por exemplo, consultas o QoL-Ad é preferível, por ser consideravelmente mais curto (13 itens) e comprovado por meio da comparação utilizando Teste t de Student. Por outro lado, quando se pretende obter avaliações mais aprofundadas, o DQoL é mais indicado por abranger mais dimensões.

Este estudo de revisão possui limitações, como a restrição do idioma (português e inglês) podendo ter sido excluídos estudos em outros idiomas, a utilização de três bases de dados e não ter realizado a busca manual o que pode ter restringido os achados na literatura.

\section{CONCLUSÃO}

Conforme o propósito desta pesquisa, a análise das propriedades de validade e consistência interna (confiabilidade) dos instrumentos destinados a avaliar a qualidade de vida do idoso com Alzheimer podem 
ser consideradas satisfatórias. A consistência interna dos instrumentos foi avaliada por meio do cálculo de alfa de Cronbach. O processo de validação dos instrumentos incluiu, em sua maioria, três domínios distintos: validade de conteúdo (tradução e retrotradução), validade de critério (concorrente e/ou convergente) e validade de construto. $O$ resultado obtido neste estudo forneceu mais uma evidência para fortalecer a noção apontada pelos autores acerca da dependência entre as dimensões da qualidade de vida dos idosos e a doença de Alzheimer. Maior desenvolvimento científico nesta área poderá, no futuro, possibilitar o planejamento e a avaliação de intervenções junto a idosos a partir das evidências baseadas sempre em instrumentos de mensuração válidos e precisos diante a doença de Alzheimer.

\section{REFERÊNCIAS}

1. Brasil. Secretaria de Atenção à Saúde. Departamento de Atenção Básica [Internet]. Envelhecimento e Saúde da Pessoa Idosa. Brasília: Ministério da Saúde; 2011[acesso em: 20 março 2016]. Disponível em:

http://bvsms.saude.gov.br/bvs/publicacoes/abcad19.pdf.

2. The WHOQOL Group. The World Health Organization quality of life assessment (WHOQOL): position paper from the World Health Organization. Social Science and Medicine. 1995; (10):1403-1409.

3. Seidl EMF, Zannon CMLC. Qualidade de vida e saúde: aspectos conceituais e metodológicos. Cadernos de Saúde Pública. 2004; 20(2):580-588.

4. Machado JC. Doença de Alzheimer. In: Freitas EV, PY L. Tratado de Geriatria e Gerontologia; 2011. p. $178-201$.

5. World Health Organization. Dementia: a public health priority [Internet]. Geneva:WHO;2012 [acesso em: 20 março 2016. Disponível: http://apps.who.int/iris/bitstream/10665/75263/1/9789241564458 eng.pdf?ua=1.

6. Keszei AP, Novak M, Streiner DL. Introduction to health measurement scales. Journal of Psychosomatic Research. 2010;(68):319-323.

7. Cozby P. Métodos de pesquisa em ciências do comportamento. São Paulo: Atlas, 2003.

8. REPPOLD CT, GURGEL LG, HUTZ CS. O processo de construção de escalas psicométricas. Avaliação Psicológica. 2014;13(2):307-310.

9. BRASIL. Conselho Federal de Psicologia (CFP). Avaliação psicológica: diretrizes na regulamentação da profissão Brasília, 2010:196.

10. Souza MT, Silva MD, Carvalho R. Revisão Integrativa: O que é e como fazer. Einstein, São Paulo. 2010; 8(1):102106.

11. Fineout-Overholt E, Levin RF, Melnyk BM. Strategies for Advancing Evidence-Based Practice in Clinical Settings. Journal of the New York State Nurses Association. 2004/2005;p.28-32.

12 Mendes KDS, Silveira RCCP, Galvão CM. Revisão integrativa: método de pesquisa para a incorporação de evidências na saúde e na enfermagem. Rev. Texto \& Contexto. 2008; 17(4):758-764.

13. The Joanna Briggs Institute. Reviewers' manual: 2014 edition. Adelaide: JBI; 2014. Disponível em: http://joannabriggs.org/assets/docs/sumari/reviewersmanual-2014.pdf.

14. BRASIL. Conselho Federal de Psicologia (CFP). Definição e regulamentação do uso, da elaboração e da comercialização de testes psicológicos- Brasília, 2003.

15. Whittemore R. Combining the evidence in nursing research: methods and implications. Nursing Research.2005;(54):56-62.

16. Moher D, Liberati A, Tetzlaff J, Altman DG. Preferred reporting items for systematic reviews and meta-analyses: the PRISMA statement.[Internet]. 2009; 6(7). Disponível em:

http://journals.plos.org/plosmedicine/article?id=10.1371/journal.pmed.1000097.

17. Ursi ES, Galvão CM. Prevenção de lesões de pele no perioperatório: revisão integrativa da literatura. Rev LatinoAmericana de Enfermagem.2006; 14(1):124-31.

18. Schölzel-Dorenbos CJM, Arons AMM, Wammes JJG, Rikkert MGMO, Krabbe PFM. Validation study of the prototype of a disease-specific index measure for health-related quality of life in dementia. Health and Quality of Life Outcomes.2012;10:118. 
19. Trigg R, Skevington SM, Jones RW. How Can We Best Assess the Quality of Life of People With Dementia? The Bath Assessment of Subjective Quality of Life in Dementia (BASQID). The Gerontological Society of America. 2007; 47(6): 789-797.

20. Wolak A, Novella JL, Drame M, Guillemin F, Di Pollina L, Ankri J, et al. Transcultural adaptation and psychometric validation of a French-language version of the QoL-AD. Aging\& Mental Health.2009; 13(4):593-600.

21. Matsui T, Nakaaki S, Murata Y, Sato J, Shinagawa Y, Tatsumi H, et al. Determinants of the Quality of Life in Alzheimer's Disease Patients as Assessed by the Japanese Version of the Quality of Life -Alzheimer's Disease Scale. Dement Geriatr Cogn Disord. 2006;21(1):182-191.

22. Gómez-Gallego M, Gómez-Amorb J, Gómez-Garcíac J. Validation of the Spanish version of the QoL-AD scale in Alzheimer disease patients, their carers, and health professionals. Neurología. 2011;27(1):4-10.

23. Novelli MPC, Nitrinia R, Caramellia P. Validation of the Brazilian version of the quality of life scale for patients with Alzheimer's disease and their caregivers (QOL-AD). Aging \& Mental Health.2010; 14(5):624-631.

24. Salas BL, Logsdon RG, Olazara'n J, Martınez-Martın P, Msu-Adru. Psychometric properties of the Spanish QoL-AD with institutionalized dementia patients and their family caregivers in Spain. Aging \& Mental Health. 2011;15(6):775783.

25. Hoe J, Katona C, Roch B, Livingston G. Use of the QOL-AD for measuring quality of life in people with severe dementia-the LASER-AD study. Age and Ageing 2005; 34(1): 130-135.

26. Voigt-Radloff S, Leonhart R, Schutzwohl M, Jurjanz L, Reuster T, Gerner A, et al. Dementia quality of life instrument - construct and concurrent validity in patients with mild to moderate dementia. European Journal of Neurology. 2012; 19(1): 376-384.

27. Novelli MMPC, Rovere HHD, Nitrini R, Caramelli P. Cross-cultural adaptation of the quality of life assessment scale on alzheimer disease. Arquivo Neuropsiquiatria. 2005;63(2-A):201-206.

28. Adánez GP. Procedimientos de construcción y análisis de tests psicométricos. In:Wechsler SM \& Guzzo RSL (Orgs.), Avaliação psicológica: Perspectiva internacional. São Paulo: Casa do Psicólogo.1999: 57-100.

29. França $A B$. Escala meta cognitiva para idosos: elaboração dos itens e análise dos parâmetros psicométricos [dissertação]:Universidade Federal de São Carlos/UFSCAR;2013.p. 139

30. International Test Commission - ITC. Diretrizes para o uso de testes [Internet]. [Acesso em: 15 de fevereiro 2016]. Campinas. 2003. Disponível em: https://www.intestcom.org/files/guideline_test_use_portuguese_brazil.pdf 31. Brod M, Stewart AL, Sands L, Walton P. Conceptualization and measurement of quality of life in dementia: the dementia quality of life instrument (DQoL). Gerontologist.1999; 39(1):25-35.

32. Inouye K, Pedrazzani ES, Cristina S, Pavarini I, Toyoda CY. Qualidade de vida do idoso com doença de Alzheimer : estudo comparativo do relato do paciente e do cuidador. 2010; 18(1):1- 8.

33. Wolak-Thierrya A, Novellaa JL, Barbea C, Morronea I, Mahmoudia R, Jollya D. Comparison of QoL-AD and DQoL in elderly with Alzheimer's disease. Aging \& Mental Health. 2015; 19(3): 274-278. 